\title{
The emerging role of gamma-delta $(\gamma \delta)$ t cells in neurological diseases and rasmussen encephalitis
}

\begin{abstract}
Consisting of gamma delta heterodimers, $\gamma \delta \mathrm{T}$ cells are inflammatory cells that play a pivotal role in a number of autoimmune neurological diseases. This review considers the emerging role of $\gamma \delta \mathrm{T}$ cells in neurological diseases having epilepsy components such as Rasmussen's encephalitis (RE), and cortical dysplasia (CD), along with other neurological conditions such as high grade gliomas, infection-induced encephalitis, multiple sclerosis (MS) and ischemic stroke.
\end{abstract}

All these neurological diseases have $\gamma \delta \mathrm{T}$ cell involvement which suggests that therapies directed toward gamma delta $\mathrm{T}$ cells with immunotherapeutics may have an important role in the treatment of these conditions including seizures and stopping disease progression, and that following these cells could be used as biomarkers of certain neurological diseases.
Volume 3 Issue 3 - 2016

\author{
Gary W Mathern,' Nazli Albayrak² \\ 'Department of Neurosurgery, University of California Los \\ Angeles, USA \\ ${ }^{2}$ School of Medicine, Acibadem University, Turkey
}

\author{
Correspondence: Nazli Albayrak, School of Medicine, \\ Acibadem University, Turkey, Istanbul,
} Email albayraknazli@gmail.com

Received: October 30, 2016 | Published: December 30, 2016

Keywords: immunotherapeutics, encephalitis, gliomas, heterodimers, rasmussen's encephalitis, dysplasia

Abbreviations: TCR, $\gamma \delta \mathrm{T}$ receptor; RE, rasmussen's encephalitis; $\mathrm{CD}$, cortical dysplasia; APCs, antigen presenting cells; MS, multiple sclerosis; BBB, blood brian barrier; GBM, glioblastoma multiforme; MGMT, methylguanine methyltransferase; NK, natural killer; WNV, west nile virus RR, relapsing remitting; PP, primary progressive; SP, secondary progressive; CSF, cerebrospinal fluid

\section{Introduction}

Typically representing only a small subset of $\mathrm{T}$ cells, T-cells with a $\gamma \delta \mathrm{T}$ receptor (TCR) have become an increasingly important cell related to neurological disease. The majority of T cells have TCRs that are composed of $\alpha \beta$ glycoprotein chains, whereas $\gamma \delta \mathrm{T}$ cells consist of $\gamma$ and $\delta$ heterodimers. The gamma chain was first cloned in 1984, which led to the detection of gamma delta T cells in 1985 . Human $\gamma \delta \mathrm{T}$ cells are based on 3 main populations depending on the delta chain type. V $\delta 1$ chains are easily recognized in the intraepithelial layer of mucosal surfaces for the purpose of keeping the integrity of the tissue against damage, infection or transformation, responding to stress-antigens on the epithelial cells and producing IL-10 with little amount of IL-2,IL-4 or IFN- $\gamma .{ }^{2} \mathrm{~V} \delta 2$ chains serve as the professional antigen-presenting cells (APCs) and they represent the majority of circulating $\gamma \delta \mathrm{T}$ cells in healthy humans. ${ }^{2} \mathrm{~V} \delta 3 \mathrm{~T}$ cells are the smallest subgroup, and are mainly found in the liver of patients with leukemia and chronic viral infections. ${ }^{2}$ Since their discovery in the mid1980 s, there have been major breakthroughs in the identification of specific and unique antigens for the $\gamma \delta \mathrm{T}$ cell receptor (TCR). There has also been emerging evidence that $\gamma \delta \mathrm{T}$ cells play a major role in local immunosurveillance, thereby controlling tumorigenesis. ${ }^{3}$ Being enhanced at epithelial surfaces and the highest abundance in the gut mucosa, and appearing to respond to self-molecules that strike potential danger or stress, this group of T cells are found less commonly than the alpha beta T cells in the body. ${ }^{3}$ TCR signaling strength and Notch signaling have been proposed to control the decision making to differentiate into $\gamma \delta$ versus $\alpha \beta$ lineages. ${ }^{4}$ Development of $\gamma \delta$ T cells, unlike $\alpha \beta$ T cells, are not affected by the absence of MHC class II or $\beta 2$ micro-globulin which suggests that they do not require classic MHC restriction for development and function. ${ }^{5}$ In recent years, it has been shown that IL-17 is an essential cytokine that regulates the recruitment of neutrophils availability during acute infection, and that $\gamma \delta \mathrm{T}$ cells are an important innate source of rapid initial IL-17 production occurring without triggering any specific TCR. ${ }^{6}$ Despite production of cytokines such as IL-17, $\gamma \delta$ T cells can induce maturation of dendritic cell and B cells. Although having a small percentage in total $\mathrm{T}$ cell population, $\gamma \delta \mathrm{T}$ cells have become an important modulator of early immune responses. ${ }^{7} Y \delta \mathrm{T}$ cells are first to pass through the blood brain barrier (BBB), and are among the first immune cells in response to IL$1 \beta$ and IL-18 as pro-inflammatory cytokines. Acting as innate immune cells, they can release cytokines, particularly IL-17 without any role of TCR ${ }^{8}$ Since neuroinflammation involves both $\alpha \beta$ and $\gamma \delta \mathrm{T}$ cells, it is possible to detect $\gamma \delta \mathrm{T}$ cells in the inflamed brain tissue as was recently demonstrated. In the study of Owens et al., ${ }^{8}$ it was shown that $\alpha \beta: \gamma \delta$ ratio was 2.2 in the brain specimen, where $\alpha \beta$ : $\gamma \delta$ ratio was7.7 in the peripheral blood of the same Rasmussen encephalitis diagnosed patient thus demonstrating relatively high presence of $\gamma \delta \mathrm{T}$ cells in the inflamed brain tissue. ${ }^{8}$

Regarding the unique characteristics of $\gamma \delta \mathrm{T}$ cells, they can be used as therapeutics in some neurological diseases, whereas in some, they are markers of disease onset. This review considers the emerging role of $\gamma \delta \mathrm{T}$ cells in neurological diseases having an epilepsy component such as Rasmussen's encephalitis, cortical dysplasia, high grade gliomas, infection induced encephalitis, ischemic stroke, and multiple sclerosis.

\section{Rasmussen's encephalitis}

Rasmussen's Encephalitis (RE) is a very rare disease characterized by intense uncontrolled partial or generalized seizures and progressive atrophy in the brain as a result of uncontrolled unihemisphere neuroinflammation. As a rare disease of the nervous system, RE is usually diagnosed with Magnetic Resonance Imaging Fluid Attenuated Inversion Recovery (MRI FLAIR) imaging that reveals inflammation in one cerebral hemisphere that progresses over time. Having surgery to remove the affected cerebral hemisphere with resulting neurologic deficits is currently the only effective treatment to reduce seizure burden and halt disease progression. RE is primarily seen in younger children affecting the cerebral cortex unilaterally with inflammation. With the disease's progression, severe hemiparesis and other neurological deficits are seen related to unilateral atrophy of cerebral tissue. Seizures usually stop after surgery, however unilateral hemiparesis and cognitive deficits are left with the patients. ${ }^{9}$ 
Corticosteroids relieve symptoms for the short term. However, while treatment with tacrolimus or intravenous (i.v.) immunoglobulins may partially stabilize the conditions of the patient, they do not have any beneficial effect on the intractable seizures. ${ }^{10}$

Whereas the presence of $\mathrm{T}$ cells and activated microglia are recognized in the inflamed cerebral hemisphere, causes of the clinical symptoms, the mechanism that triggers the cells to be confined in the hemisphere is not known yet. The cytotoxic $\mathrm{T}$ cells which kill damaged cells by infection or in other ways are found in the affected hemisphere, reacting to antigens presented by neurons and astrocytes, but not the oligodendrocytes. ${ }^{11,12}$ Once it was hypothesized that antibodies against the glutamate receptor Glur3 would be the potential cause of RE. ${ }^{9}$ However these antibodies are not found in all RE cases and are also not specific to the disease. ${ }^{9}$

Since recognizing cytomegalovirus, herpes simplex virus, and Epstein Barr virus sequences in the post-operative brain specimens of RE patients, there has been no information that confirms a specific pathogen which is common for all RE cases ${ }^{8}$. As there are no known pathogens affecting all the RE cases, and antibodies are not found in all RE cases, it is most likely RE is an autoimmune disease. ${ }^{9}$

A recent study found that gamma-delta T-cells are involved early in the course of RE, ${ }^{9}$ patients diagnosed with RE that had undergone surgery within a shorter time from the disease onset than the other group of patients taking longer time for surgery have different levels of IFN- $\gamma$ and CXCL9 mRNAs detected in the brain specimens. IFN- $\gamma$ is a cytokine that has a pivotal role in innate and adaptive immunity against viral, some bacterial and protozoal infections. Abnormal IFN- $\gamma$ expression might be associated with several auto inflammatory and autoimmune diseases, and CXCL9 is a T-cell chemo attractant which is induced by IFN- $\gamma{ }^{13}$ There were higher levels of mRNAs found in the patient specimens that were taken in the shorter time to surgery. This study advanced the authors to the idea of having pronounced Th1 immune response in the early phase of the disease that declines 1 to 2 years in the disease course. Since the higher levels of IFN- $\gamma$ in cerebrospinal fluid in the earlier stages of RE was reported by Takahashi et al., ${ }^{14}$ detection of large numbers of CD4+ and CD8+ lymphocytes from the same specimen from which the high levels of IFN- $\gamma$ were detected, are consistent with an initial Thl response.

In a recent study of Owens et al., ${ }^{8}$ there was involvement of both $\alpha \beta T$ cell and non-classical $\gamma \delta \mathrm{T}$ cell in RE cases. Y $\delta \mathrm{T}$ cells are restricted to the area where they can recognize the persistent infection which might be the cause of RE or if the cause is self-antigens, they recognize the self-antigen associated with stressed cells. ${ }^{8}$

In RE cases, $\gamma \delta \mathrm{T}$ cells can be considered as both the marker of the disease as well as the indicators of the choice of therapy for future practice. As it was emphasized in the study of Owens et al. ${ }^{8}$ that the existence of IFN- $\gamma$ and CXCL9, where IFN- $\gamma$ is the product of $\gamma \delta \mathrm{T}$ cell and CXCL9 is the chemo attractant of these particular cells; it was suggestive of $\gamma \delta \mathrm{T}$ cells being a marker of RE.

\section{Cortical dysplasia}

Cortical dysplasia (CD) is a neurodevelopmental disorder related to abnormal cell proliferation and differentiation before birth associated with refractory epilepsy. ${ }^{15}$ In order to treat epilepsy of the patient diagnosed with $\mathrm{CD}$, surgical removal is advanced with neuro imaging techniques. ${ }^{16}$ A clearer definition of the classification of the disease has helped to have a better understanding of the basic mechanism of CD. In the study of Owens et al., ${ }^{8}$ sequencing $\delta 1$ chain PCR fragments revealed that the same prevalent third complementarity determining region (CDR3) sequences, the most diverse of CDRs which are heavy and light chains of antibodies, were found in all of the brain specimens and were even detected in brain tissue from 15 focal cortical dysplasia cases. The study of Owens et al., ${ }^{8}$ indicates that the presence of the identical $\delta 1$ subtype clones in CD cases advances the involvement of a common inflammatory pathway in both $\mathrm{CD}$ and RE. Although the mechanism of epileptogenesis in CD is not completely understood, there is an involvement of interactions of different cell types, and the mechanism does not work simply due to the imbalance in GABAergic/ glutamartergic neurotransmissions. ${ }^{16}$

\section{High grade gliomas}

Glioblastoma Multiforme (GBM) is a very aggressive glioma that originates from star-shaped glial cells that support the health of nerve cells within the brain. Referred to as a grade IV astrocytoma by the World Health Organization (WHO), GBMs are the most invasive and rapidly growing, commonly spreading type of glial tumors. According to the American Association of Neurological Surgeons (AANS), in 2015, there were 15,320 cases resulting in death. GBM can also arise "de novo"or upgrade from lower-grade astrocytomas or oligodendrogliomas. With an incidence of 3.19 in 100,000 adults per year, GBM accounts for $45.2 \%$ of malignant primary brain tumors, $54 \%$ of all gliomas, and $16 \%$ of all primary brain and CNS tumors. ${ }^{17}$ Treatment of the high grade gliomas, in particular GBM, still remains a great challenge in neuroncology. Since average mean survival is 12-15months for GBM diagnosed patients, the only standard therapy that is offered so far is a combination of radiotherapy, chemotherapy, and surgery.

Studies of Dieli et al., ${ }^{18}$ and Todaro $\mathrm{M}$ et al., ${ }^{19}$ showed that $\gamma \delta \mathrm{T}$ cells have anti-tumoral activities in vivo on malignant brain tumors, and Fujimiya et al. mentioned that GBM cell lines can be killed by $\gamma \delta \mathrm{T}$ cells in vitro and tumor progression can be reduced in vivo.

Cancer immunotherapy was accepted by the Science editorial board as a Breakthrough of the Year 2013 and approved as the development of and evolving new pattern in cancer treatment. The studies that have been done so far related to immunotherapy in brain tumors have shown success rates in experimental models. However due to the brain's protected environment and the immunosuppressive effect of the tumor, there has been little success in clinical trials. According to the study of Nakazawa et al., ${ }^{20}$ there are different cytotoxic effects of $\gamma \delta \mathrm{T}$ cells on GBM cell lines. In order to achieve a better anti-tumoral effect, enhancing the cytotoxic effect on GBM cells has a key role in therapy. Zoledrante (ZOL), an inhibitor of osteoclastic activity, is known to stimulate peripheral blood-derived $\gamma \delta \mathrm{T}$ cells and sensitize tumors to $\gamma \delta \mathrm{T}$ cell-mediated killing. Since $\gamma \delta \mathrm{T}$ cells kill only low numbers of GBM cells, ZOL enhances allogeneic $\gamma \delta \mathrm{T}$ cell-mediated killing. Results of the study done by Nakazawa et al., ${ }^{20}$ Demonstrate that allogeneic $\gamma \delta \mathrm{T}$ cell-mediated immunotherapy would be safe and a novel strategy for human GBM. Y $\delta \mathrm{T}$ cells are unable to penetrate human GBM parenchyma causing low numbers of GBM cells to be killed, however, ZOL can penetrate the BBB-disrupted GBM tumors without any modification. This suggests that using ZOL and $\gamma \delta \mathrm{T}$ cells in combination may provide more successful immunotherapy for GBM diagnosed patients.

Not requiring any peptide recognition and priming and not being constrained by the selectivity and restriction of the MHC, unlike $\alpha \beta T$ cells, $\gamma \delta \mathrm{T}$ cells have a rapid secretion of chemokines and cytokines as well as target lysis. Notably, $\gamma \delta \mathrm{T}$ cells recognize stress-induced NKGD2D ligands such as MICA/B and UL-16 binding proteins expressed by GBM but not adjacent normal brain tissues. ${ }^{21,22}$ The anti-tumor cytotoxicity of $\gamma \delta \mathrm{T}$ cells is partially associated with the innate immunity of the mentioned ligands. ${ }^{22}$ Although expression 
of stress molecules on tumor cells can sensitize the recognition of the tumor cell by $\gamma \delta \mathrm{T}$ cells as an innate effector, surrounding normal tissue would also be affected by the natural toxicities due to the proinflammatory environment.

Temozolomide (TMZ) is a principle chemotherapeutic agent used to treat GBM. The activating receptor Natural-Killer group 2, member $\mathrm{D}(\mathrm{NKG} 2 \mathrm{D})$ and its ligands play an important role in immune response to tumors. ${ }^{23}$ NKG2D ligands on TMZ- resistant high grade gliomas, TMZ enables $\gamma \delta \mathrm{T}$ cell to recognizethe tumor cell to lyse. Since TMZ is also very toxic for $\gamma \delta \mathrm{T}$ cells, in the study of Lamb et al., ${ }^{22}$ it was shown that genetic modification using lentoviral vector encoding DNA repair enzyme $\mathrm{O}(6)$-alkylguanine DNA alkytransferase (AGT) from the $\mathrm{O}(6)$-methylguanine methyltransferase(MGMT) cDNA, offered resistance. Without impairing the anti-tumoral action of $\gamma \delta \mathrm{T}$ cells, TMZ can be combined with immunotherapy to treat GBM.

\section{Infection induced encephalitis}

Natural Killer (NK) and $\gamma \delta \mathrm{T}$ cells are both innate lymphocytes that have a rapid and unspecific action on viral infections and other pathogens. It has also been demonstrated that they can make a unique link between innate and adaptive immunity. ${ }^{24}$

Scalise et al., ${ }^{25}$ conducted a study between eight children with acquired Toxoplasmosis gondii infection and a control group of ten children. It was reported that the children with acquired toxoplasmosis gondii during their acute phase of the illness had higher number of $\gamma \delta \mathrm{T}$ cells in their bloodstream than the control group. The great majority of the lymphocytes found in the bloodstream were BB3+. Since BB3+ cells can bear the T cell marker CD45RO on their membrane, they can respond to nominal antigens as proliferating, therefore it suggests that they are engaged to combat many human pathogens.

Having NK and $\gamma \delta \mathrm{T}$ cells effects on viral infections and other pathogens, there are studies showing the distinct roles of both NK and $\gamma \delta$ T cells in control of West Nile virus (WNV) infection. WNV is an important health issue in United States. It is, a mosquito-born, single stranded RNA flavivirus having symptoms including fever, headache, myalgia, meningitis, and encephalitis. In the study of Wang et al., ${ }^{26}$ it was demostrated that TCR $\beta$-deficient hosts have higher mortality suggesting that $\alpha \beta T$ cells have a major contribution to survival. Y $\delta \mathrm{T}$ cells are also involved in the WNV infection as a part of immune response. Y $\delta \mathrm{T}$ cells expand rapidly in the spleen and peritoneal cavity early in infection. Y $\delta \mathrm{T}$ cells regulate memory $\mathrm{t}$ cell development by promoting dendritic cell maturation and activation. ${ }^{26}$ For a target population, due to the unique role of both NK and $\gamma \delta \mathrm{T}$ cells in association with dendritic cells in host innate and adaptive immunity, the studies provide important insight to the immunotherapy in $\mathrm{WNV}^{24}$

\section{Multiple sclerosis}

Multiple Sclerosis (MS) is a chronic autoimmune disease characterized by demyelination of neuronal axons in CNS. MS was classified as relapsing remitting (RR), primary progressive (PP) or secondary progressive (SP) depending upon the clinical presentations of the disease (Gandhi et al., 2010). Infiltration of self-reactive immune cells from peripheral circulation to CNS has a major role in the inflammation in MS. Since MS is a complex disease with components of both genetic and environmental triggers, dysregulation of immune homeostasis and tolerance is inevitable. In the immunology of MS, auto-reactive T lymphocytes directed against myelin proteins are thought to have a major role in pathogenesis of MS. A study of Stinissen et al., ${ }^{27}$ demonstrated that there was a marked response to the vaccine and production of high levels of IL-2 and that TNF- $\alpha$ and IL-10, $\gamma \delta \mathrm{T}$ cells have an immunoregulatory role in $\mathrm{MS}^{27}$
In the experimental autoimmune encephalomyelitis (EAE) model, an animal model of MS disease in mice, it was shown by Wohler et al. that trafficking of $\gamma \delta \mathrm{T}$ cells occured prior to the onset of disease in acute EAE. When they induced the disease, $\gamma \delta \mathrm{T}$ cells were found immediately after the transfer of the cells to the mouse brains (Wohler et al. 2010). The exact role of $\gamma \delta \mathrm{T}$ cells in MS is not well understood. In EAE, they have diverse effects such as initiating the pathogenesis, or showing protection or may have no effect on disease. With the mechanism of heat-shock protein, expressed by oligodendrocytes, $\gamma \delta \mathrm{T}$ cells can lyse these particular cells (Freedman et al., 1991). Y $\delta \mathrm{T}$ cells can be seen in high number in MS patients with active or progressive disease, and contribute to disease pathology by exerting direct cytotoxic effect on oligodendrocytes (Gandhi et al. 2011). Since infiltration of $\mathrm{T}$ cells with myelin reactivity expressing a prominent role of T cells in MS, there is an involvement of myelin-specific CD4+ T cell. ${ }^{28}$ Th1 and Th17 cells, as two major pathogenic effector CD4+ $\mathrm{T}$ cell subsets, are the main source of IFN- $\gamma$. The production of both IFN $-\gamma$ and TNF- $\alpha$ cytokines by $\gamma \delta$ T cells are needed for acute EAE. These results indicate that $\gamma \delta \mathrm{T}$ cells are critical in setting the stage for the development of EAE and also play a role in immunoregulation in MS. In summary, innate immune cells act in either a beneficial or harmful fashion in MS.

\section{Ischemic stroke}

Stroke, including brain infarction, is a leading cause of death and disability worldwide, however no successful therapy has been established that can be initiated at the subacute phase of brain infarction. ${ }^{29}$ Since in EAE model Th17 cells have been in both the onset and the progression, most studies have been focused on Th17 cells. ${ }^{29}$ In the study of Adamski et al., ${ }^{30}$ there was an increase of IL-17 observed, although the number of $\gamma \delta \mathrm{T}$ cells in stroke patients is was almost $50 \%$ decreased. Y $\delta \mathrm{T}$ cells are one of the major resources of IL-17 and are directly activated by IL-23. These lymphocytes, which produce IL-17 but not IFN- $\gamma$, are referred as IL-17 producing $\gamma \delta \mathrm{T}$ cells. Recent studies have shown that the number of IL-17 producing $\gamma \delta \mathrm{T}$ cells peak in the ischemic brain tissue 3-5days after the injury, and that the infarct volume evolves day 3-5 after onset in humans suggesting that IL-17 producing $\mathrm{T}$ lymphocytes have a role in the infarct growth. ${ }^{30-32}$

\section{Possible role of gamma delta $t$ cells in epilepsy and RE}

Epilepsy is a chronic neurological disease based on recurring seizures and accompanied by cognitive deficits and mood disorders in common, affecting $1 \%$ of the human population worldwide. ${ }^{33}$ There is a positive feedback cycle between brain inflammation and epileptogenesis due to recurrent seizures and brain injuries that leads to stimulation of proinflamatory cytokines. ${ }^{33}$ Studies done so far have suggested that inflammation in the brain might advance epileptogenesis, ${ }^{33}$ however protective immune responses are also found in epilepsy besides other neurological diseases such as multiple sclerosis. ${ }^{33}$

Emphasizing the detection of an increasing number of auto antibodies in serum and Cerebrospinal fluid (CSF) of patients with new onset drug-resistant focal epilepsy, Xu et al., ${ }^{33}$ also stressed the association of RE with T-cell activation and production of proinflammatory cytokines by activated glia. Effector CD8+ cytotoxic $\mathrm{T}$ lymphocytes are proffered to induce astrocytic and neuronal apoptosis and degeneration, one of the distinguishing characteristics of RE. ${ }^{12}$

\section{Conclusion}

Consisting of heterodimers, $\gamma \delta \mathrm{T}$ cells play a critical role in local immunosurveillance. In this review, the link between neurological 
diseases with a special emphasis on epilepsy and $\gamma \delta \mathrm{T}$ cells are explained. Since $\gamma \delta \mathrm{T}$ cells are found in autoimmune disorders, high grade gliomas, infection-induced encephalitis and ischemic stroke, these cells are in wide-spectrum. Because it is possible to recognize $\gamma \delta \mathrm{T}$ cells in the inflamed area of the brain due to the disease progress, having them isolated from that particular area, and using therapeutics against the disease prognosis might suggest further studies.

For RE, further considerations should be established on genetic and environmental interactions. Although surgery is indicated in the treatment of RE, in order to overcome with the post-operative neurological deficits, further studies are needed to elucidate the efficacy of new non-surgical treatment options to control the seizures while preserving neurological functions. For future studies with long-term outcome data are needed to have effective wide spectrum comparative analysis. The RE Children's Project promotes collection of tissues from patient's with RE available for research studies. ${ }^{10}$ As it is reported in the study of Thilo et al., ${ }^{34}$ a lady was treated with rituximab after having Rasmussen's encephalitis for seven years. For the first time she was seizure-free for several months since her diagnosis. In order to preserve neurological function and delay the cerebral hemiatrophy, tacrolimus-an inhibitor of cytokine synthesis- is used for the treatment, although it has no role on seizure outcome. ${ }^{35-38}$

In high grade gliomas, although the importance of immunotherapy is revealed by several studies, some practical issues still need addressing featuring an investigation of optimal scheduling and dosage for both irradiation and immunotherapy ${ }^{21}$ Further studies are strongly needed to determine the optimal parameters and test the safety of $\gamma \delta \mathrm{T}$ cells in clinical settings for aggressive brain tumor treatment.

There are studies suggesting the role of immunotherapy in infection-induced encephalitis. Increased knowledge on the host responses on this particular type of encephalitis, future studies may advance the development of vaccines and immunotherapy and with a better understanding of immunopathogenesis of the disease. Future studies of WNV depend on the spread of the virus and the prevalance in the future.$^{26}$ Immunotherapy in clinical use in patients with acute encephalitis and the relevant viral or antibody biomarkers has changed clinical outcomes. Development of appropriate animal models or in vitro trials may render it eligible to study both the causes and the effects of particular diseases and might advance direct clinical trials. ${ }^{10}$

In multiple sclerosis, the role of the innate immune system can be either beneficial or detrimental. Since the aspect of innate immunity and the role of $\mathrm{T}$ cells are better defined, it should render it possible to design a therapy that has immuno-pathogenic innate cells altered as immuno-regulatory immune cells. Having more knowledge about the molecular mechanism of $\gamma \delta \mathrm{T}$ cells and their link between different phases of autoimmune diseases would suggest future studies on development of novel immunotherapeutics based on $\gamma \delta \mathrm{T}$ cells. ${ }^{5}$

Ischemic stroke has an increased amount of IL-17 $\gamma \delta$ T cells which are activated by IL-23. As the major source of IL-17 and IFN- $\gamma$, having reduction in number of $\gamma \delta \mathrm{T}$ cells in CAD suggests possible therapeutic options. ${ }^{30}$

\section{Acknowledgments}

We would like to express our sincere appreciation to Geoffrey Owens and Thabiso Chirwa for their immense contributions to various drafts of the review. Additionally, we thank the external peer reviewers for their valuable comments that have enriched the the review.

\section{Conflicts of interest}

Author declares there are no conflicts of interest.

\section{Funding}

None.

\section{References}

1. Weintraub BC, Hedrick SM. The enigmatic specificity of gamma delta T cells. Immunol Res . 1995;14(3):163-175.

2. Wu YL, Ding YP, Tanaka Y, et al. $\gamma \delta \mathrm{T}$ Cells and their potential for immunotherapy. Int J Biol Sci. 2014;10(2):119-135.

3. Girardi M. Immunosurveillance and immunoregulation by $\gamma \delta$ T cells. $J$ Invest Dermatol. 2006;126(1):25-31.

4. Washburn T, Schweighoffer E, Gridley T, et al. Notch activity influences the alphabeta versus gammadelta $\mathrm{T}$ cell lineage decision. Cell. 1997;88(6):833-843.

5. Paul S, Shilpi, Girdhari L. Role of gamma-delta T cells in autoimmunity. J Leukoc Biol. 2015;97(2):259-271.

6. Kabelitz D, Déchanet Merville J. Editorial: Recent advances in gamma/ delta $T$ cell biology: new ligands, new functions, and new translational perspectives. Front Immunol. 2015;6: 371.

7. Patil RS, Bhat SA, Dar AA, et al. The Jekylland Hydestory of IL17producing $\gamma \delta$ T cells. Front Immunol. 2015;6:37.

8. Owens GC, Erikson KL, Malone CC, et al. Evidence for the involvement of gamma delta $\mathrm{T}$ cells in the immune response in Rasmussen's encephalitis. J Neuroinflammation. 2015;12:134.

9. Owens GC, Huynh MN, Chang JW, et al. Differential expression of interferon-gamma and chemokine genes distinguishes Rasmussen enceohalitis from cortical dysplasia and provides evidence for an early Th1 immune respone. J Neuroinflammation. 2013;10: 56.

10. Varadkar S, Bien CG, Kruse CA, et al. Rasmussen's encephalitis: clinical features, pathobiology, and treatment advances. Lancet Neurol . 2014;13(2):195-205.

11. Bien CG, Bauer J, Deckwerth TL, et al. Destruction of neurons by cytotoxic T cells: a new pathogenic mechanism in Rasmussen's encephalitis. Ann Neurol . 2002;51(3):311-318.

12. Bauer J, Elger CE, Hans VH, et al. Astrocytes are a specific immunological target in Rasmussen's encephalitis. Ann Neurol. 2007;62(1):67-80.

13. Schroder K, Hertzog PJ, Ravasi T, et al. Interferon- $\gamma$ : an overview of signals, mechanisms and functions. J Leukoc Biol . 2004;75(2):163-189.

14. Takahashi Y, Mine J, Kubota Y, et al. A substantial number of Rasmussen syndrome patients have increased IgG, CD4+ T cells, TNF alpha, and Granzyme B in CSF. Epilepsia. 2009;50(6):1419-1431.

15. Hauptman JS, Mathern GW. Surgical treatment of epilepsy associated with cortical dysplasia: 2012 update. Epilepsia. 2012;53(Suppl 4):98-104.

16. Abdijadid S, Mathern GW, Levine MS, et al. Basic mechanisms of epileptogenesis in pediatric cortical dysplasia. CNS Neurosci Ther. 2015;21(2):92-103.

17. Thakkar JP, Dolecek TA, Horbinski C, et al. Epidemiologic and molecular prognostic review of glioblastoma. Cancer Epidemiol Biomarkers Prev. 2014;23(10):1985-1986.

18. Dieli F, Vermijlen D, Fulfaro F, et al. Targeting human cdT cells with zoledronate and interleukin-2 for immunotherapy of hormone refractory prostate cancer. Cancer Res . 2007;67(15):7450-7457.

19. Todaro M, D'Asaro M, Caccamo N, et al. Efficient killing of human colon cancer stem cells by cdT lymphocytes. J Immunol. 2009; 182(11):7287-7296.

20. Nakazawa T, Nakamura M, Park YS, et al. Cytoxic human peripheral blood-derived gamma delta $\mathrm{T}$ cells kill glioblastoma cell lines: implications for cell-based immunotherapy for patients with glioblastoma. J Neurooncol . 2014;116(1):31-49. 
21. Pereboeva L, Harkins L, Wong S, et al. The safety of allogeneic innate lymphocyte therapy for glioma patients with prior with cranial irradiation. Cancer Immunol Immunother. 2015;64(5):551-562.

22. Lamb Jr LS, Bowersock J, Dasgupta A, et al. Engineered drug resistant gamma delta $\mathrm{T}$ cells kill giloblastoma cell lines during a chemotherapy challenge: A strategy for combining chemo- and immunotherapy. Plosone. 2013;8(1):e51805.

23. Nausch N, Cerwenka A. NKG2D ligands in tumor immunity. Oncogene. 2008;27(45):5944-5958.

24. Wang T, Welte T. Role of Natural Killer and Gamma delta T cells in West Nile virus infection. Viruses. 2013;5(9):2298-2310.

25. Scalise F, Gerli R, Castellucci G, et al. Lymphocytes bearing the $\gamma \delta \mathrm{T}$ cell receptor in acute toxoplasmosis. Immunol. 1992;76:668-670.

26. Wang T, Fikrig E. Immunity to west nile virus. Curr Opin Immunol . 2004;16(4):519-523.

27. Stinissen P, Zhang J, Vandevyver C, et al. Gammadelta T cell responses to activated $\mathrm{T}$ cells in multiple sclerosis patients induced by $\mathrm{T}$ cell vaccination. J Neuroimmunol . 1998;87(1-2): 94-104.

28. Mastorodemos V, Ioannou M, Panayotis V. Cell-based modulation of autoimmune responses in multiple sclerosis and experimental autoimmune encephalomyelitis: therapeutic implications. Neuroimmunomodulation. 2015;22(3):181-195.

29. Shichita T, Sugiyama Y, Ooboshi H, et al. Pivotal role of cerebral interleukin-17-producing $\gamma \delta \mathrm{T}$ cells in the delayed phase of ischemic brein injury. Nat Med . 2009;15(8): 946-950.
30. Adamski MG, Li Y, Wagner E, et al. Pre-existing hypertension dominates $\gamma \delta \mathrm{T}$ cell reduction in human ischemic stroke. Plos one. 2014;9(5):e97755

31. Jensen KD, Su X, Shin S, et al. Thymic selection determines gammadelta $T$ cell effector fate: antigen-navie cells make interleukin-17 and antigeneperienced cells make interferon gamma. Immuniy. 2008;29(1):90-100.

32. Nakamura $R$, Shibata $K$, Yamada $H$, et al. Tyk2-signaling plays an important role in host defense against escherichia coli through Il-23-induced Il-17 production by $\gamma \delta \mathrm{T}$ cells. $J$ Immunol . 2008;181(3):2071-2075.

33. Xu D, Miller SD, Koh S. Immune mechanisms in epileptogenesis. Front Cell Neurosci . 2013;7:195.

34. Thilo B, Stingele R, Knudsen K, et al. A case of Rasmussen encephalitis treated with rituximab. Nat Rev Neurol. 2009;5(8):458-462.

35. Mastrangelo M, Mariani R, Menichella A. Eponym:Rasmussen Syndrome. Eur J Pediatr. 2010;169(8):919-924.

36. Chen Z, Freedman MS. Correlation of specialized CD16+ gammadelta $\mathrm{T}$ cells with disease course and severity in multiple sclerosis. $J$ Neuroimmunol. 2008;194(1-2):147-152.

37. Lamb LS. Gamma delta cells as immune effectors against high-grade gliomas. Immunol Res. 2009;45(1):85-95.

38. Rasmussen T, Olszewski J, Lloydsmith D. Focal seizures due to chronic localized encephalitis. Neurology. 2015;8(6):435-445. 